\title{
Molecular Determinant of Regioselective Hydroxylation of Docetaxel by CYP3A4
}

\author{
Bernard Monsarrat ${ }^{1}$, Odile Thoison ${ }^{2}$, Joelle Dubois ${ }^{2}$ and Thierry Cresteil ${ }^{2}$ \\ ${ }^{1}$ IPBS, CNRS UMR 5089, 205 route de Narbonne, 31077 Toulouse cedex, and ${ }^{2}$ ICSN, CNRS \\ UPR 2301, avenue de la Terrasse, 91198 Gif sur Yvette cedex, France.
}

\begin{abstract}
The metabolism of taxanes by human liver microsomes is regioselective: the major metabolite of paclitaxel formed by CYP2C8 results from the hydroxylation on the taxane ring at C-6. Hydroxylations on the lateral chain at $\mathrm{C}-13$ are catalyzed by CYP3A4 on either the tert-butyl of docetaxel or the C-3' phenyl of paclitaxel. Furthermore, the presence of the acetyl group in position 10, has been shown to play an important role in determining the regioselective oxidation by CYP. The biotransformation of a series of docetaxel analogues by human liver microsomes and recombinant CYP expressed in HEK293 cells was examined by high-performance liquid chromatography/mass spectrometry. The formation of derivatives was lost when the tertbutyl of docetaxel was replaced by an ethyl group. Addition of an aliphatic chain at either position 7 or 10 led to the formation of oxidized metabolites, whereas addition at both 7 and $\mathbf{1 0}$ totally impaired the production of derivatives. Similarly, the insertion of a phenylbenzoyl group in position 10 prevented the biotransformation of the molecule. The site of hydroxylation could be clearly located on the aliphatic chain inserted in position 10, but remained on the tert-butyl at $\mathrm{C}-13$ when the aliphatic chain was added in position 7 . These reactions were catalyzed only by CYP3A4: recombinant CYP3A4 generated the same metabolites as liver microsomes did, whereas neither CYP3A5 nor 2C8 or 2C9 could form derivatives. In conclusion, the presence of substituents in position 10 of the docetaxel molecule plays a pivotal role in determining the site of oxidation by CYP3A4.
\end{abstract}

Received 03/16/09; accepted 05/06/09

Correspondence: Dr. Thierry Cresteil, ICSN, CNRS UPR 2301, Avenue de la terrasse, 91198 Gif sur Yvette, cedex, France

Tel. 33-169 823 643, Fax. 33169823643

email:cresteil@icsn.cnrs-gif.fr
Keywords: Paclitaxel; Docetaxel; CYP3A4; CYP2C; Biotransformation; Human liver

\section{Introduction}

The hydroxylation of taxanes by cytochromes P450 (CYP)1 has been shown to be regioselective in relation with the location and nature of substituents on the taxane ring. As shown in the first reports on paclitaxel metabolism by human liver microsomes, the major derivative was hydroxylated in position 6 on the taxane ring and was formed by CYP2C8, whereas a minor metabolite was oxidized on the C-3' phenyl group of the lateral chain by CYP3A4 (1-2). The biotransformation of docetaxel was rather simple since a single primary derivative resulted from an oxidation on the tert-butyl of the lateral chain further cyclized to give two secondary metabolites. Both CYP3A4 and 3A5 were capable to realize this reaction but displayed different affinities (3-4). No oxidation occurred on the taxane ring of docetaxel and CYP2C8 was totally inactive in the biotransformation of docetaxel (5).

Based on these regioselective oxidations by CYP2C8 and CYP3A4, further investigations have demonstrated that the presence of an acetyl group in position 10 could substantially modified the pattern of biotransformation and the apparent affinity of taxanes for CYP. Thus the addition of an acetyl group in position 10 of docetaxel partly restored the hydroxylation on the taxane ring by CYP2C8 whereas the loss of the acetyl group from the paclitaxel molecule did not alter the pattern of biotransformation but significantly reduced the affinity of 10-deacetylpaclitaxel for CYP2C8 and CYP3A4 (5). This clearly attributed a pivotal role in the biotransformation process of taxane molecules to the substituent in position 10 .

Another feature was the difference in the regioselective oxidation of taxanes by $\mathrm{CYP} 2 \mathrm{C} 8$ and CYP3A4. It became evident that the hydroxylation at the C-6 of the taxane ring of paclitaxel, 10- 
deacetylpaclitaxel and 10-acetyldocetaxel was only due to CYP2C8. However the hydroxylation of baccatin III and 10-deacetylbaccatin III presumably in position 6 was not catalyzed by CYP2C 8 but by CYP3A4. This would indicate that the presence of the lateral chain at $\mathrm{C}-13$ was implicated in the orientation of the taxane molecule into the active site of CYP3A4: in the absence of the lateral chain, the taxane ring orientated to place the C- 6 in the vicinity of the heme, in contrast with molecules having a hydrophobic lateral chain at $\mathrm{C}-13$ which placed the tert-butyl or the phenyl group close to the heme.

Basically the hydroxylation of taxanes by CYP conducted to a drastic reduction of the

cytotoxic capacity of the parent molecule: thus the 6hydroxypaclitaxel is more than 30 -fold less

potent than paclitaxel in the in vitro inhibition of cell proliferation (6-8). Similarly the hydroxylated metabolite of docetaxel showed poor in vitro cytotoxicity in P388 cells and was inefficient to reduce the tumor growth in B16 tumor-bearing mice (9).

The knowledge of biotransformation pathways undergone by taxanes has a double interest.

First by addition of substituent on the native docetaxel molecule, it is possible to change the site of hydroxylation and to modulate the intensity of reaction to improve the therapeutic efficiency of the administered drug. Second, the use of substituted taxanes could allow exploring the orientation of substrates into the active site of CYP3A4 and constituted a useful tool to probe the hydrophobic pocket of this enzyme.

In the present study, we have used docetaxel analogues differently substituted with aliphatic chains in position 7,10 or in both positions. Incubations were run with human liver microsomes and with recombinant CYP3A4, 3A5, 2C8 and CYP2C9. Results clearly indicated that CYP3A4 is the major isoform involved in docetaxel biotransformation and that substitutions on the docetaxel molecule considerably modify the orientation of the substrate into the active site of CYP3A4.

\section{Materials and Methods Synthesis of docetaxel analogues}

Docetaxel was provided by Aventis (Antony, France). Its analogues were synthesized as previously reported (10-11) and their structures were depicted in Figure 1. All commercial chemicals were from the highest available grade.

\section{Microsome preparation}

Human hepatic tissues were collected from organ donors according to the recommendations of the local ethical committee. Samples were obtained within the hour following death and immediately frozen in liquid nitrogen before be kept at $-80^{\circ} \mathrm{C}$ until use. Microsomes were prepared as stated previously (12). The total cytochrome P450 and protein content were estimated as reported elsewhere (1).

Expression of recombinant human $\mathbf{P 4 5 0}$

HEK293 cells were cultured in Dulbecco's modified Eagle's medium supplemented with $10 \%$ fetal calf serum at $37^{\circ} \mathrm{C}$ under $5 \%$ CO2. The preparation of stable transfectants expressing CYP2C8, 2C9, 3A4 and 3A5 in HEK293 cells was detailed previously (5).

\section{Metabolism of docetaxel analogues}

Stock solutions of docetaxel and its analogues were prepared in methanol at a concentration of $10 \mathrm{mM}$. Microsomal proteins corresponding to 0.3 nmoles of total P450 were incubated with $50 \mu \mathrm{M}$ docetaxel or its analogues for $30 \mathrm{~min}$ at $37^{\circ} \mathrm{C}$ in the presence of a NADPH generating system (5). A blank was incubated in the same conditions but without NADPH. After three extractions by dichloromethane, organic phases were pooled and evaporated to dryness under nitrogen. The residues were dissolved in 200 $\mathrm{\mu l}$ of acetonitrile/water (70:30, v:v) and analyzed by HPLC and HPLC/MS.

When incubated with cell lines, $50 \mu \mathrm{M}$ docetaxel or its analogues were added to $4 \mathrm{ml}$ of culture medium without fetal calf serum in $75 \mathrm{~cm} 2$ tissue culture flasks containing cells at near confluence.

Incubations were carried out with cell lines stably transfected with CYP and compared with the native HEK293 expressing no CYP. After $24 \mathrm{hrs}$ at $37^{\circ} \mathrm{C}$, cells were scraped and the cells and culture medium were extracted with $5 \mathrm{ml}$ dichloromethane three times. Organic phases were pooled and evaporated under nitrogen before be analyzed by HPLC.

\section{Characterization of docetaxel metabolites}

The metabolites of docetaxel and its analogues were resolved by reverse phase HPLC. The following conditions were used: i) Ultrasphere ODS column 250x4.6 mm (Beckman Coulter Inc, Fullerton, CA) or Hypurity C18, $5 \mu \mathrm{m}, 150 \times 4.6 \mathrm{~mm}$ (Thermo, San Jose, CA), ii) a mixture of water/acetonitrile operated as linear gradients from $30 \%$ to $60 \%$ acetonitrile for docetaxel and from 60 to $100 \%$ for 
analogues with hydrophobic substituents. Elution was realized at a flow rate of $1 \mathrm{ml} / \mathrm{min}$. iii) UV detection with a diode array detector. Microsome and cell extracts were dissolved in $100 \mathrm{\mu l}$ methanol and $10 \mu \mathrm{l}$ were injected onto the HPLC column. To characterize the structure of docetaxel and docetaxel analogue metabolites, we used on line HPLC-MS (TSQ 700 ThermoFinnigan, San Jose, CA) with an atmospheric pressure chemical ionization-mass spectrometry interface mode as reported elsewhere $(5,13)$ or Surveyor LC system coupled with LCQ deca ion trap spectrometer (ThermoFinnigan, San Jose, CA). The LCQ deca is equipped either with ESI or APCI probe. The operating parameters for ESI were: spray needle voltage $4 \mathrm{kV}$, nitrogen sheath gas (80 arbitrary units), nitrogen auxiliary gas (20 arbitrary unit), capillary temperature $250^{\circ} \mathrm{C}$; for APCI conditions were: nitrogen sheath gas (80 arbitrary units), capillary temperature $250^{\circ} \mathrm{C}$, APCI vaporizer temperature $450^{\circ} \mathrm{C}$. The LCQ deca was operated alternatively in positive or negative ion mode. ESILCMS gave exclusively quasi molecular ion $[\mathrm{M}+\mathrm{Na}]+$ for all compounds whereas positive or negative APCI-LCMS gave data on fragmentation.

\section{Results \\ Metabolism of docetaxel and its analogues by human liver microsomes}

In a first attempt the metabolism of docetaxel by human liver microsomes was verified in our conditions. As previously shown, several peaks corresponding to the oxidation product and its cyclized derivatives were formed in vitro. Then, analogues were incubated in the same conditions, extracted and analyzed by HPLC. Respective to their chemical structure and lipophilicity, different elution procedures have been used to efficiently separate docetaxel analogues from their derivatives. In 3'-de-tert-butyl 3'ethyldocetaxel, the $t$-butyl group at C-3' was replaced by an ethyl group (10). When this compound was incubated with liver microsomes and NADPH no metabolite was formed in vitro.

Substitutions were made in position 7 , by addition of an aliphatic chain of either 4 (7-

butanoyldocetaxel) or 9 carbons (7nonanoyldocetaxel). The addition of an aliphatic chain resulted in the occurrence of a prominent if not only metabolite with a shorter retention time than the parent molecule: elution times were 14.3 min versus $20.6 \mathrm{~min}$ for 7-butanoyldocetaxel, and $22.4 \mathrm{~min}$ vs $27.2 \mathrm{~min}$ for 7-nonanoyldocetaxel (Figure 2). Additionally, two very minor peaks were

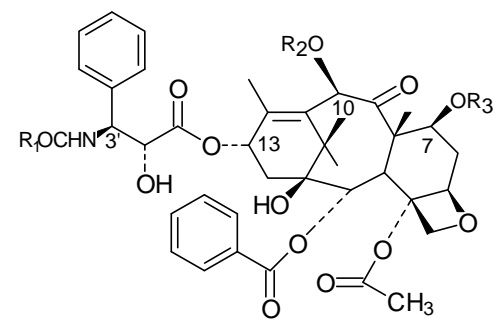

$\begin{array}{lccc} & \mathrm{R} 1 & \mathrm{R} 2 & \mathrm{R} 3 \\ \text { docetaxel } & \mathrm{OC}\left(\mathrm{CH}_{3}\right)_{3} & \mathrm{H} & \mathrm{H} \\ \text { 10-butanoyldocetaxel } & \mathrm{OC}\left(\mathrm{CH}_{3}\right)_{3} & \mathrm{OC}\left(\mathrm{CH}_{2}\right)_{2} \mathrm{CH}_{3} & \mathrm{H} \\ \text { 10-octanoyldocetaxel } & \mathrm{OC}\left(\mathrm{CH}_{3}\right)_{3} & \mathrm{OC}\left(\mathrm{CH}_{2}\right)_{6} \mathrm{CH}_{3} & \mathrm{H} \\ \text { 10-nonanoyldocetaxel } & \mathrm{OC}\left(\mathrm{CH}_{3}\right)_{3} & \mathrm{OC}\left(\mathrm{CH}_{2}\right)_{7} \mathrm{CH}_{3} & \mathrm{H} \\ \text { 10-biphenyldocetaxel } & \mathrm{OC}\left(\mathrm{CH}_{3}\right)_{3} & \mathrm{OC}\left(\mathrm{C}_{6} \mathrm{H}_{4}\right)\left(\mathrm{C}_{6} \mathrm{H}_{5}\right) & \mathrm{H} \\ \text { 7-butanoyldocetaxel } & \mathrm{OC}\left(\mathrm{CH}_{3}\right)_{3} & \mathrm{H} & \mathrm{OC}\left(\mathrm{CH}_{2}\right)_{2} \mathrm{CH}_{3} \\ \text { 7-nonanoyldocetaxel } & \mathrm{OC}\left(\mathrm{CH}_{3}\right)_{3} & \mathrm{H} & \mathrm{OC}\left(\mathrm{CH}_{2}\right)_{7} \mathrm{CH}_{3} \\ \text { 7,10-dibutanoyldocetaxel } & \mathrm{OC}\left(\mathrm{CH}_{3}\right)_{3} & \mathrm{OC}\left(\mathrm{CH}_{2}\right)_{2} \mathrm{CH}_{3} & \mathrm{OC}\left(\mathrm{CH}_{2}\right)_{2} \mathrm{CH}_{3} \\ \text { 7,10-diheptanoyldocetaxel } & \mathrm{OC}\left(\mathrm{CH}_{3}\right)_{3} & \mathrm{OC}\left(\mathrm{CH}_{2}\right)_{5} \mathrm{CH}_{3} & \mathrm{OC}\left(\mathrm{CH}_{2}\right)_{5} \mathrm{CH}_{3} \\ \text { 7,10-dinonanoyldocetaxel } & \mathrm{OC}\left(\mathrm{CH}_{3}\right)_{3} & \mathrm{OC}\left(\mathrm{CH}_{2}\right)_{7} \mathrm{CH}_{3} & \mathrm{OC}\left(\mathrm{CH}_{2}\right)_{7} \mathrm{CH}_{3} \\ \text { 3'de-t-butyl-3'ethyldocetaxel } & \mathrm{OCH} \mathrm{CH}_{3} & \mathrm{H} & \mathrm{H}\end{array}$

Figure 1. Chemical structure of docetaxel and its substituted analogues

monitored at 10.7 and 12 min with 7 butanoyldocetaxel.

Similarly, substitutions were made in position 10 by addition of an aliphatic ester of 4,8 or 9 carbons or a $p$-phenylbenzoyl group. With 10butanoyldocetaxel as substrate, two peaks were eluted with retention times of 11.1 and $11.4 \mathrm{~min} v \mathrm{~s}$

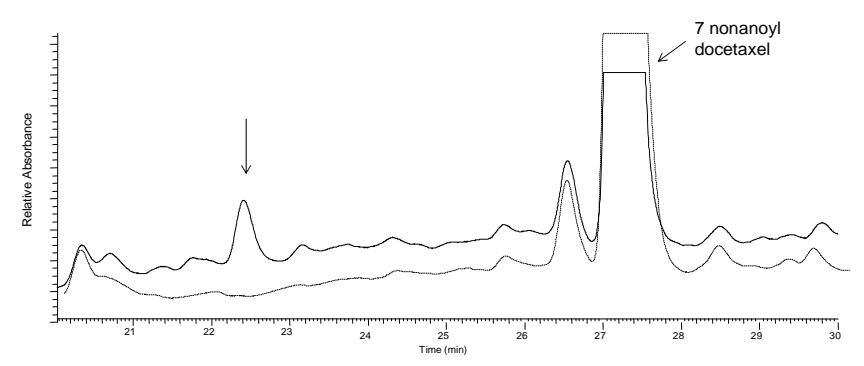

Figure 2. HPLC chromatogram of 7-nonanoyldocetaxel and its metabolite generated by CYP3A4 expressing Ad 293 cells. Arrow refers to metabolite formed by CYP3A4 eluting at 22.4 min versus $27.2 \mathrm{~min}$ for unchanged 7-nonanoyldocetaxel. Dash line: native HEK293 cells and solid line: HEK293-CYP3A4 cells.

$18 \mathrm{~min}$ for the unchanged 10-butanoyldocetaxel, as well as three peaks were monitored with 10 octanoyldocetaxel at $9.5, \quad 10.0$ and $10.6 \mathrm{~min}$ compared with $20.3 \mathrm{~min}$ for the native molecule. With 10-nonanoyldocetaxel four peaks were 
monitored at 11.5, 12.0, 12.3 and 12.6 min vs 23.3 min for the parent molecule (Figure 3). In contrast no derivative was observed when a $p$-phenylbenzoyl chain was inserted in position 10 . When a butanoyl-, heptanoyl- or nonanoyl- side chain was inserted simultaneously in positions 7 and 10, no product with a reduced retention time was observed suggesting that no metabolite was formed in the presence of NADPH by liver microsomes.

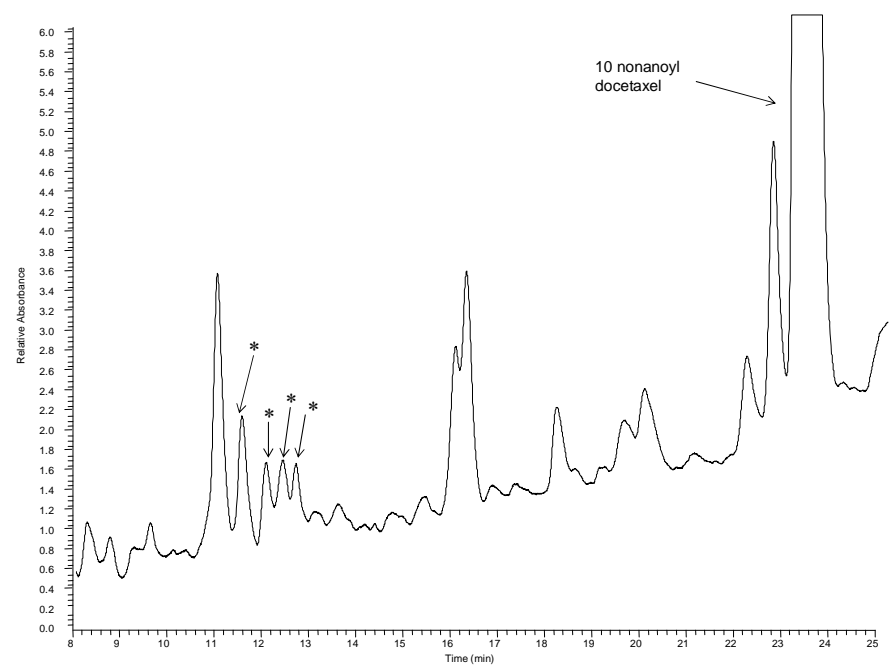

Figure 3. HPLC chromatogram of 10-nonanoyldocetaxel and its metabolites generated by CYP3A4 expressing cells. Arrows and asterisks refer to metabolites formed by HEK293-CYP3A4 cells and absent from native HEK293 cells.

\section{Metabolism of docetaxel analogues by recombinant CYP}

Then we focused on molecules actively metabolized by human liver microsomes to identify the CYP isoform responsible for the formation of derivatives. Docetaxel analogues were incubated in HEK293 cells devoid of monooxygenase activities or in HEK293 cells expressing either CYP2C8, 2C9, $3 \mathrm{~A} 4$ or $3 \mathrm{~A} 5$. The only isoform capable to carry out the reactions was CYP3A4 in a similar manner than microsomes did. CYP3A5 an isoform which hydroxylated the $t$-butyl of docetaxel with a lower efficiency than CYP3A4 had no visible effect on docetaxel analogues. Neither CYP2C8, nor CYP2C9 were capable to produce derivatives of docetaxel and its analogues. Thus only CYP3A4 was responsible for the biotransformation of 7-butanoyl-, 7-nonanoyl-, 10-butanoyl-, 10-octanoyl- and 10-nonanoyldocetaxel occurring with microsomal preparations.
Characterization of metabolites of 10-butanoyl-, 10-octanoyl-and 10-nonanoyldocetaxel

To tentatively characterize the structure of metabolites formed during incubation of 10butanoyl-, 10-octanoyl- and 10-nonanoyldocetaxel with liver microsomes, analysis was performed in ESI- and APCI-LCMS. Figure 4A shows the fragmentation pattern obtained with positive APCILCMS of the unchanged 10-butanoyldocetaxel. The parent ion of the intact 10-butanoyldocetaxel in positive mode $[\mathrm{M}+\mathrm{H}]+$ was $m / z 878$ and fragments included $\mathrm{m} / z 822$ (- tert-butyl), 790 (- side chain at C-10), 734 (-tert-butyl - side chain at C10), 690 (COO - tert-butyl - side chain at C-10), 597 (- side chain at $\mathrm{C} 13$ ) and 182 (lateral chain at C-13 - tertbutyl -COO). The two peaks eluting at 11.1 and 11.4 min had the same parent ion $[\mathrm{M}+\mathrm{H}]+$ at $\mathrm{m} / z 894$ suggesting a hydroxylated product of the parent molecule. Their fragmentation in the positive mode
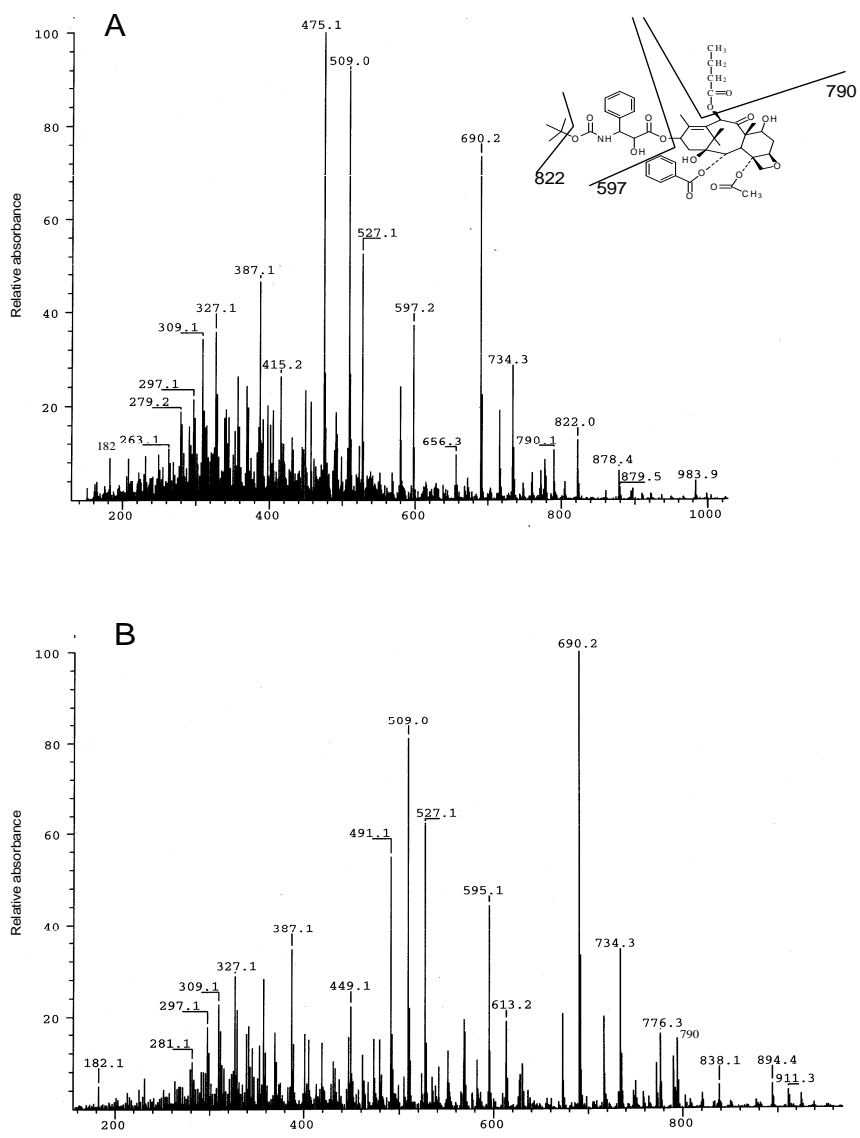

(Figure 4B) was identical with unchanged fragments

Figure 4. APCI-LC/MS positive mode spectrum of 10butanoyldocetaxel $(A)$ and its metabolite $(B)$ eluting at $11.1 \mathrm{~min}$. 
at $182,690,734$ and 790 indicated that oxidation does not occur on the taxane ring or the lateral chain at C-13 and was confirmed by the shift from $\mathrm{m} / \mathrm{z} 822$ to 838 and 597 to 613 locating the oxidation site on the lateral chain at C-10. Furthermore, the positive ESI-LCMS of 10-butanoyldocetaxel and its metabolites gave a prominent quasi molecular ion peak at $m / z 900$ and $916[\mathrm{M}+\mathrm{Na}]+$ respectively confirming their molecular mass (data not shown).

When 10-octanoyldocetaxel was incubated with liver microsomes in the presence of NADPH, three peaks were resolved and eluted between 9.5 and 10.6 min. Investigated separately by LC-MS (ESI and APCI), the material present in the three peaks gave identical fragmentation profiles. The positive ESI-LCMS showed characteristic quasi molecular ion peak at $\mathrm{m} / z 956[\mathrm{M}+\mathrm{Na}]+$ for the unchanged 10octanoyldocetaxel and quasi molecular ion peak at $m / z 972[\mathrm{M}+\mathrm{Na}]+$ for its metabolites showing a shift of its molecular mass of +16 amu compatible with a monohydroxylation of the molecule. The 10octanoyldocetaxel displayed in positive APCI-LCMS a parent ion $[\mathrm{M}+\mathrm{H}]+$ at $m / z 934$ and characteristic fragments at 878 (- tert butyl), 834 (- tert butyl COO), 790 (- side chain at C-10), 734 ( - tert-butyl side chain at $\mathrm{C}-10), 653$ (- side chain at $\mathrm{C}$-13) and 182 (side chain at C-13 -tert-butyl - COO) (Figure 5). Fragments of the three metabolites generated in APCI-LCMS were identical and indicated that the addition of the oxygen atom did not occur on the side chain at C-13 and its tert-butyl $(\mathrm{m} / z$ 894, 850 and $669)$ but was likely associated with the side chain at $\mathrm{C}-10$ with an intact taxane $\operatorname{ring}(\mathrm{m} / z$ 790, 734). The similarities between the fragmentation profiles of the three metabolites suggested that they are isomers but did not allow locating precisely the site of oxidation.

Moreover when the aliphatic chain was longer like in 10-nonanoyldocetaxel, four products (Figure 3) were generated with an additional +16 amu of their molecular mass in ESI-LCMS suggesting a monohydroxylation of the parent molecule. The four compounds displayed identical fragmentation patterns in APCI-LCMS suggesting that all four metabolites were isomers. In APCI negative mode, the unchanged 10-nonanoyldocetaxel had characteristic fragments at 788 (-side chain at C-10), 280 (side chain at C-13) and 157 (side chain at C-10). The four metabolites retained typical fragments at $\mathrm{m} / \mathrm{z} 788$ and 280 indicating that oxidation did not occur on the lateral chain at C-13 or the taxane ring, but rather on the aliphatic chain at C-10 and was confirmed by the shift of the fragment containing the aliphatic chain from 157 to 173 (Figure 6). Thus, when a substitution was made on carbon 10 with an aliphatic chain, this chain is the site of a single oxidation by CYP 3A4, while no reaction occurs on the tert-butyl group of the lateral chain at C-13.
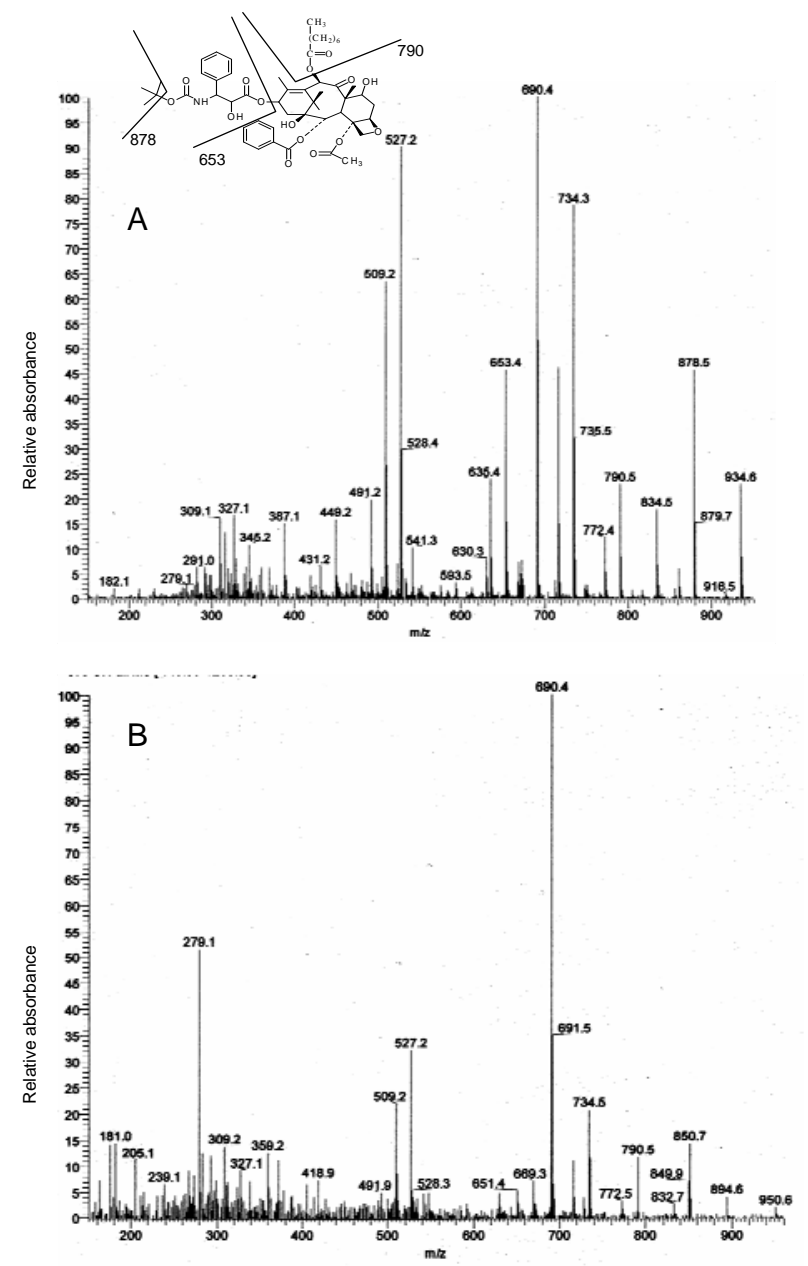

Figure 5. APCI-LC/MS, positive mode spectrum of 10octanoyldocetaxel (A) and its metabolite (B) eluting at $9.5 \mathrm{~min}$.

\section{Characterization of metabolites of 7-butanoyl- and 7-nonanoyldocetaxel}

Similar ESI and APCI-LCMS experiments were performed with analogues of docetaxel modified in position 7. As for 10-butanoyldocetaxel, a major peak with a shorter retention time was resolved when 7-butanoyldocetaxel was incubated with human liver microsomes or CYP3A4-expressing cell line. The parent ion of the intact 7butanoyldocetaxel was similar to that of the 10butanoyldocetaxel with $[\mathrm{M}+\mathrm{Na}]+m / z 900$ in ESILCMS, and the parent ion of its derivative was identical to that of the derivative of 10 - 
butanoyldocetaxel with $m / z 916[\mathrm{M}+\mathrm{Na}]+$ in ESILCMS. Analysis of fragments in APCI-LCMS of 7 butanoyl- and 10-butanoyldocetaxel showed similar pattern with special emphasis on fragments characteristic of the loss of the $\mathrm{C}-13$ side chain in positive mode $(\mathrm{m} / z 597)$ and the $\mathrm{C}$-13 side chain in negative mode $(\mathrm{m} / z 280)$. In the major oxidation product of 7-butanoyldocetaxel, $\mathrm{m} / z$ fragments were 597 and 296 indicating that the site of oxidation was on the lateral chain at C-13. The two minor peaks eluting at 10.7 and $12.0 \mathrm{~min}$ exhibited a different fragmentation pattern although their parent ion $[\mathrm{M}+\mathrm{Na}]+$ was similar at $\mathrm{m} / z 916$. Both compounds had the $m / z$ fragments in APCI-LCMS at 613 and 280 suggesting that oxidation was not on the lateral chain at C-13, and the $\mathrm{m} / z$ fragment at 790 indicating that oxidation was located on the lateral chain at C-7 (data not shown). However the very low amount of product formed during incubation has made the characterization difficult.
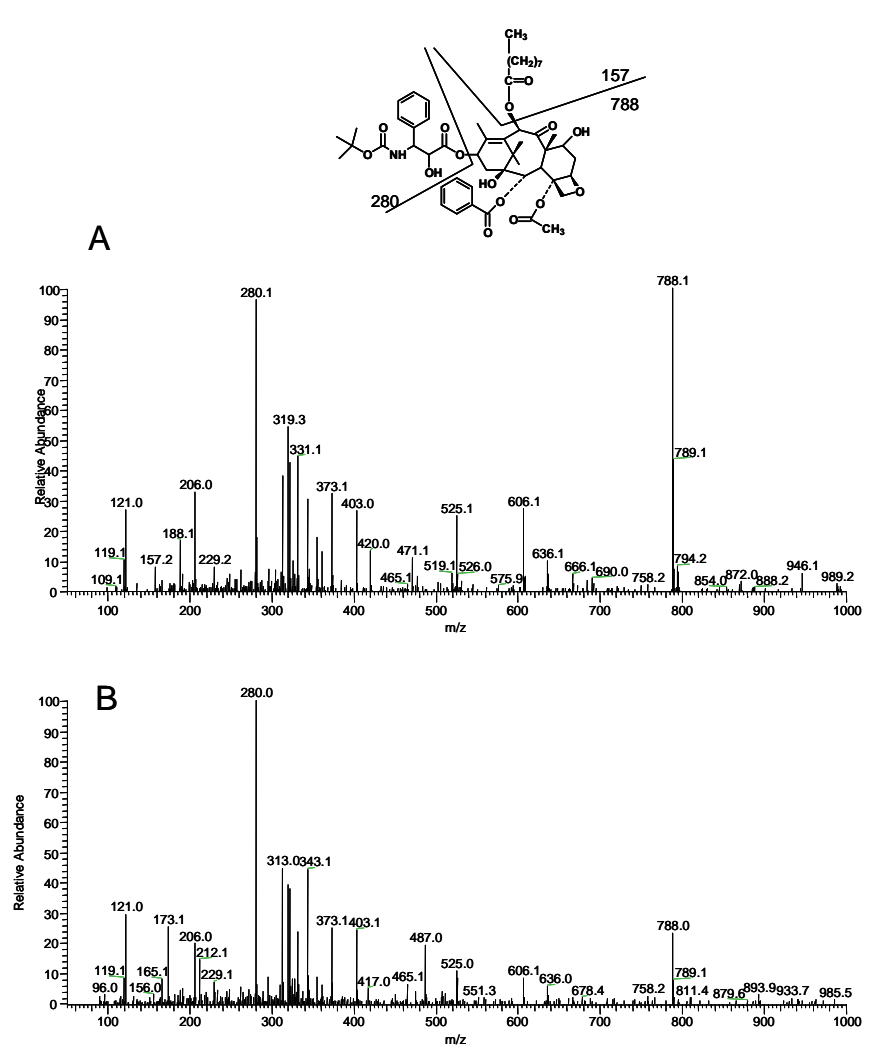

Figure 6. APCI-LC/MS, negative mode of 10-nonanoyldocetaxel (A) and its metabolite (B) eluting at $11.5 \mathrm{~min}$.

With 7-nonanoyldocetaxel, the unique peak observed was eluted at $22.4 \mathrm{~min}$ (Figure 2) and displayed in ESI-LCMS a molecular mass at +16 $\mathrm{amu}, \mathrm{m} / z 986(\mathrm{M}+\mathrm{Na})+$ compatible with a single oxidation of the parent molecule. Once again, the fragmentation in APCI-LCMS of 7nonanoyldocetaxel was identical to that of 10nonanoyldocetaxel. Figure 7 showed the fragments at 157 (lateral chain at C-7) and 280 (lateral chain at $\mathrm{C}$-13) in the unchanged 7-nonanoyldocetaxel molecule shifting to 157 and 296 in the oxidized compound. Thus, the hydroxylation was clearly located on the lateral chain at C-13. Thus modifications of docetaxel made on the $\mathrm{C}-7$ atom did not modify the hydroxylation pattern of docetaxel on the side chain at C-13.
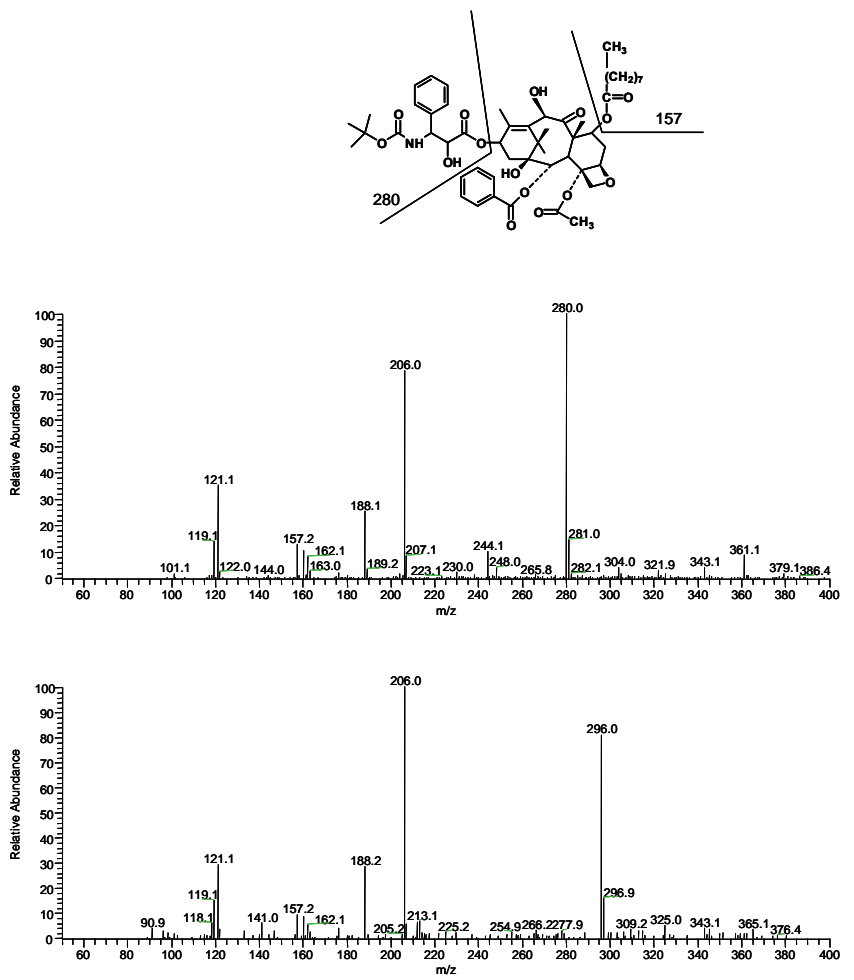

Figure 7. APCl-LC/MS, negative mode $\mathrm{m} / \mathrm{z}(50-400]$ of $7-$ nonanoyldocetaxel $(A)$ and its metabolite $(B)$ eluting at $22.4 \mathrm{~min}$.

Biotransformation rate of docetaxel analogues

When $50 \mu \mathrm{M}$ docetaxel or its analogues were incubated with liver microsomes, the extent of the oxidation reaction depended on the structure of the lateral chain at C10 irrespective of the site of hydroxylation. Thus with docetaxel $1.8 \mathrm{nmol}$ of substrate were oxidized on the tert-butyl in $30 \mathrm{~min}$ by $1 \mathrm{nmol}$ P450 (Table 1). When an acetyl group was added in position 10, 3.5 nmoles were oxidized on the tert-butyl. The addition of a butyl or an octyl group in position 10 orientated the oxidation to the lateral chain in 10 and the rates of oxidation were slightly increased to 4.2 and $5.7 \mathrm{nmol}$ by $1 \mathrm{nmol}$ 
P450 in 30 min. With the bulky $p$-phenylbenzoyl chain in position 10 of docetaxel no formation of derivatives was observed.

\section{Discussion}

We have shown previously that substitutions on the paclitaxel and docetaxel molecules could orientate the metabolism of the molecule and determine the CYP isoform involved in the reaction. Basically, the presence of a side chain at C-13 has two consequences: first the oxidation on the side chain at C-13 occurs at either the tert-butyl of docetaxel or the phenyl group of paclitaxel $(1,3)$. Second, the oxidation on the side chain is exclusively supported by CYP3A4 while the oxidation on the taxane group in position 6 is restricted to CYP2C8. However, when the side chain in position 13 is missing, the metabolism on the taxane ring (likely in position 6) is realized by CYP3A4 (5). Therefore, docetaxel is only oxidized by CYP3A4 (and to a lesser extent by CYP3A5) on its tert-butyl group. The addition of an acetyl group in position 10 of docetaxel markedly modifies the pattern of hydroxylation: in addition to the oxidation on the tert-butyl, CYP3A4 also oxidizes the 3'-phenyl group of the side chain with a high affinity (5). To go further in the structure-activity relationship we have designed docetaxel analogues to test the hydrophobic and steric hindrance acceptable by CYP and investigated the site of oxidation.

In a first approach, we examined the presence of potential derivatives by HPLC in relation with the structure of docetaxel analogues. The substitution of the tert-butyl of the side chain by an ethyl group abolishes the oxidation on the side chain by liver microsomes in the presence of NADPH. The addition of an aliphatic chain in position 10 leads to the formation of one or several derivatives by human liver microsomes. Consistent with the former observation that the addition of an acetyl group increased the rate of transformation of docetaxel, the presence of a butanoyl or an octanoyl group stimulated the formation of derivatives to reach $3.5 \%$ of transformation in $30 \mathrm{~min}$. When investigated in mass spectrometry, derivatives are oxidation products on the side chain at C-10. No oxidation on the tert-butyl group was noticed and consequently, no cyclization product was detected. This clearly indicates that the addition of an aliphatic chain in position 10 prevents the tert-butyl to be close enough to the heme and totally impair its oxidation and its further cyclization. Alternatively, the insertion of an aliphatic chain at at C-10 in the Table 1. Biotransformation of $50 \mu \mathrm{M}$ taxane by
human liver microsomes

Taxane

Total metabolites

(nmole/nmoles CYP/30 min)

$\begin{array}{ll}\text { 10-deacetylpaclitaxel } & 1.33 \\ \text { paclitaxel } & 6.66 \\ \text { docetaxel } & 1.83 \\ \text { 10-acetyldocetaxel } & 3.5 \\ \text { 10-butanoyldocetaxel } & 4.2 \\ \text { 10-octanoyldocetaxel } & 5.7 \\ 10-p \text {-phenylbenzoyldocetaxel } & <0.15 \\ 7,10 \text {-diheptanoyldocetaxel } & <0.15\end{array}$

docetaxel molecule orientates the molecule into the active site and places this chain in the vicinity of the heme, instead of the tert-butyl group. This implicates a substantial reorientation of the substrate in the heme pocket. The number of oxidation products on the lateral chain at $\mathrm{C} 10$ is depending on the length of the aliphatic chain from two regioisomer metabolites for the butanoyl chain up to four isomers with the nonanoyl chain. This suggests that the chain is flexible enough to allow different conformations in the vicinity of the heme conducting to several sites of hydroxylation.

Unexpectedly, when a $p$-phenylbenzoyl group was introduced at C-10, no reaction is observed: this could be interpreted as a steric hindrance of the lateral chain preventing the correct insertion of the docetaxel molecule in the vicinity of the catalytic heme. It could be noted that a $p$-phenylbenzoyl group is almost as long as a nonanoyl linear chain but is very rigid and cannot fold up. Then it can be assumed that 10-nonanoyldocetaxel is metabolized because the $\mathrm{C}-10$ side chain is folded. The different locations of hydroxylation on this flexible chain may likely reflect different folding.

Similarly, aliphatic chains have been inserted in position 7 on the docetaxel molecule. As shown with insertion on aliphatic chain at C-10, insertion at C-7 led to the formation of derivatives hydroxylated by human CYP. However, in contrast with analogues substituted in position 10, the presence of an aliphatic chain in position 7 did not reorientate the docetaxel molecule into the active site of the enzyme and led only to metabolite hydroxylated in majority, if not exclusively, on the lateral chain at C-13. When two aliphatic chains were inserted at C-7 and C-10, we cannot detect the formation of any reaction products. Several hypotheses could account to explain this lack of metabolism: first the presence of two chains in position 7 and 10 might increase the 
size of the molecule and prevent its entry and its correct orientation into the CYP pocket. Second the hydrophobicity of aliphatic chains might be too high and prevent the entry of the molecule into the heme pocket. Whatever the cause, the consequence is the impairment of the substrate penetration into the active site or its correct position in the vicinity of heme.

The major, if not unique, CYP isoform involved in the biotransformation of docetaxel and its analogues is CYP3A4. It has been shown previously that CYP3A5 was also capable to oxidize the tertbutyl of docetaxel with a lower affinity (4), whereas the addition of an acetyl group in position 10 restores the hydroxylation on the taxane ring of 10 acetyldocetaxel by CYP2C8 (5). In the present study and as expected from data collected on the biotransformation of docetaxel, CYP2C8 and 2C9 had no action whatever the analog investigated, and CYP3A5 did not produce any derivative at a $50 \mu \mathrm{M}$ concentration of docetaxel analogues. Thus all reactions are clearly catalyzed by CYP3A4: this was confirmed by the formation of docetaxel analogue derivatives only by recombinant CYP3A4 expressed in HEK293 cells and by the examination of derivatives formed during cell incubations by HPLC/MS.

A good correlation has been reported previously between the cytotoxicity of these docetaxel analogues on $\mathrm{KB}$ cells and their capacity to inhibit in vitro the microtubule disassembly (11). Basically, analogues modified at C-7 or C-10 displayed a cytotoxic potency comparable to that of docetaxel with alkyl side chains of less than 11 carbons, as well as with the addition of a $p$-phenylbenzoyl at C10. In case of substitution at both $\mathrm{C} 7$ and $\mathrm{C} 10$, the inhibition of microtubule disassembly decreased rapidly with the length of the aliphatic chain. Furthermore, analogues bearing a long chain with hydrophilic functions retained much better biological activities than hydrophobic analogues as seen with the cytotoxic potency on KB cells and microtubule disassembly tested in vitro (11). So the location of hydrophobic area in the docetaxel molecule could markedly modify its biological activity.

For the biotransformation of docetaxel analogues, two points could be raised: first, for analogues modified at C-10, the rate of hydroxylation increased with the chain length as well as the number of metabolites formed. Second, modifications introduced at both C-7 and C-10 totally prevented the biotransformation of docetaxel analogues by
CYP3A4, even with short aliphatic chains (e.g. butanoyl chains) exhibiting a moderate hydrophobicity. Thus it seems that steric hindrance, as well as hydrophobicity, could modulate the biotransformation rate of docetaxel analogues. Several aspects could be considered from a clinical point of view: the drug should be soluble in biological fluids allowing its wide distribution in the body without use of detergents or additives, its affinity for tubulin should be high and its hepatic biotransformation should be as low as possible to increase its availability. Docetaxel analogues substituted at both 7 and 10 with short aliphatic chains fulfill these criteria and might constitute a new generation of taxane with a greater efficiency.

\section{References}

1. Cresteil T, Monsarrat B, Alvinerie P, Treluyer JM, Vieira I, Wright M. Taxol metabolism by human liver microsomes: identification of cytochrome P450 isozymes involved in its biotransformation. Cancer Res 1994;54:38692.

2. Rahman A, Korzekwa KR, Grogan J, Gonzalez FJ, Harris JW. Selective biotransformation of taxol to 6ahydroxytaxol by human cytochrome P450 2C8. Cancer Res 1994;54:5543-6.

3. Royer I, Monsarrat B, Sonnier M, Wright M, Cresteil T. Metabolism of docetaxel by human cytochromes P450: interactions with paclitaxel and other antineoplastic drugs. Cancer Res 1996;56:58-65.

4. Shou M, Martinet M, Korzekwa KR, Krausz KW, Gonzalez FJ, Gelboin HV. Role of cytochrome P450 3A4 and $3 \mathrm{~A} 5$ in the metabolism of taxotere and its derivatives: enzyme specificity, interindividual distribution and metabolic contribution in human liver. Pharmacogenetics 1998;8:391-401.

5. Cresteil T, Monsarrat B, Dubois J, Sonnier M, Alvinerie P, Gueritte F. Regioselective metabolism of taxoids by human CYP3A4 and 2C8: structure-activity relationship. Drug Metab Dispos 2002;30:438-45.

6. Harris JW, Katki A, Anderson LW, Chmurny GN, Paukstelis JV, Collins JM. Isolation, structural determination and biological activity of 6a-hydroxytaxol the principal human metabolite of taxol. J Med Chem 1994;37:706-9.

7. Kumar G, Ray S, Walle T, Huang Y, Willingham M, Self $\mathrm{S}$, Bhalla K. Comparative in vitro cytotoxic effects of taxol and its major human metabolite 6a-hydroxytaxol. Cancer Chemother Pharmacol 1995;36:129-35.

8. Sparreboum A, Huizing MT, Boesen JJB, Nooijen WJ, Van Tellingen O, Beijnen JH. Isolation, purification and biological activity of mono- and dihydroxylated paclitaxel metabolites from human faeces. Cancer Chemother Pharmacol 1995;36:299-304.

9. Vuilhorgne M, Gaillard C, Sanderink GJ, et al. Metabolism in taxoid drugs. In: Georg GI, Chen TT, Ojima 
I and Vyas DM editors. Taxane Anticancer agents: Basic science and Current status; ACS symposium 1995; series 583 p98-110.

10. Dubois J, Guenard D, Gueritte-Voegelein F, Guedira N, Potier P, Gillet B, Beloeil JC. Conformation of taxotere and analogues determined by NMR spectroscopy and molecular modeling studies. Tetrahedron 1993;49:6533-44. 11. Guénard D, Thoret S, Dubois J, Adeline MT, Wang Q, Gueritte F. Effects of the hydrophobicity of taxoids on their interaction with tubulin. Bioorg Med Chem 2000;8:145-56.

12. Cresteil T, Flinois JP, Pfister A, Leroux JP. Effect of microsomal preparations and induction on cytochrome P450-dependent monooxygenases in fetal and neonatal rat liver. Biochem Pharmacol 1979;28:2057-63.

13. Royer I, Alvinerie P, Armand JP, Ho LK, Wright M, Monsarrat B. Paclitaxel metabolites in human plasma and urine: identification of 6a-hydroxytaxol, 7-epitaxol and taxol hydrolysis products using liquid chromatography/atmospheric-pressure chemical ionization mass spectrometry. Rapid Commun Mass Spectrometry 1995;9:495-502. 